\title{
Prospective evaluation of the clinical utility of endoscopic submucosal dissection (ESD) in patients with Barrett's esophagus: a Western center experience
}

Authors

Institutions
Roxana M. Coman ${ }^{1}$, Takuji Gotoda ${ }^{2}$, Christopher E. Forsmark ${ }^{1}$, Peter V. Draganov ${ }^{1}$

'Division of Gastroenterology, Hepatology and Nutrition, University of Florida, Gainesville, Florida, United States

2 Department of Gastroenterology and Hepatology, Tokyo Medical University, Tokyo, Japan submitted

2. November 2015 accepted after revision: 23. January 2016

\section{Bibliography}

DOI http://dx.doi.org/

10.1055/s-0042-101788

Published online: 30.3.2016

Endoscopy International Open

2016; 04: E715-E721

(c) Georg Thieme Verlag KG

Stuttgart · New York

E-ISSN 2196-9736

\section{Corresponding author}

Peter V. Draganov, MD

University of Florida

Division of Gastroenterology,

Hepatology, and Nutrition

1329 SW 16th

Archer Road

PO Box 100214

Gainesville, FL 32610

dragapv@medicine.ufl.edu
Background and study aims: Endoscopic submucosal dissection (ESD) carries significant advantages over endoscopic mucosal resection. As such, ESD is an established therapy for esophageal squamous cell carcinoma but there are only limited data on ESD as therapy for Barrett's esophagus (BE). Thus, we prospectively evaluated the outcomes of ESD in patients with BE with highgrade dysplasia (HGD) and early esophageal adenocarcinoma (EAC) performed in a Western center.

Patients and methods: This is a prospective cohort study. Indications for ESD included: (1) early EAC defined as lesions with intramucosal cancer or superficial submucosal invasion; (2) early EAC with positive lateral margin after EMR; and (3) nodularity with HGD that could not be removed en-bloc with EMR

Results: From October 2013 to July 2015, 36 consecutive patients (median age 69, 32 males) un-

\section{Introduction}

The rising incidence of esophageal adenocarcinoma (EAC) over the past three to four decades has been well documented and poses a significant health burden in Western countries [1]. Barrett's esophagus (BE) is universally present in patients with EAC and the length of $\mathrm{BE}$, male sex, obesity and white race are the main established risk factors [2].

Although the global prevalence of $\mathrm{BE}$ is low $(<5 \%)$, it is higher in selected groups such as those with gastroesophageal reflux disease $(>15 \%)$ [3]. A large retrospective study in Denmark of more than 42,000 patients with $\mathrm{BE}$ revealed an annual risk of developing EAC of $0.4 \%$ [4]. Other similar population-based studies from the United Kingdom, Netherlands and Northern Ireland, as well as a meta-analysis of high-quality studies derived similar estimates of progression to EAC in patients with $\mathrm{BE}[5,6]$. High-grade dysplasia (HGD) is the derwent ESD at our center. Median procedure time was 88 minutes, with median maximal diameter of resected specimens of $49 \mathrm{~mm}$. En-bloc, R0, and curative resection rates were $100 \%, 81 \%$, and $69 \%$, respectively.

Intramucosal EAC was found in 13 patients (36\%), and submucosal invasion in 13 patients (36\%). In $59 \%$ of the cases, there was discrepancy in the pre- and post-ESD histopathologic diagnosis.

Adverse events occurred in 8 patients (22\%), including one episode of bleeding treated with endoscopy and seven esophageal strictures, which were successfully managed with dilations. Conclusions: ESD for BE with HGD/early EAC is feasible and safe with resulting very high en-bloc and $\mathrm{R} 0$ resection rates. ESD provided for more accurate pathologic evaluation and significant discrepancy between the pre- and post-ESD histopathological diagnosis was noted.

immediate precursor of EAC and, until recently, surgical esophageal resection was the therapy of choice. This approach was supported by the high progression rate of HGD to EAC and even more concerning, the high prevalence of undetected EAC in patients undergoing esophagectomy for BE with HGD [7].

With the advent of new technologies and techniques, endoscopic treatments have now been accepted as first-line therapy for BE with HGD and early EAC [8]. The rationale for endoscopic resection is that lesions confined to the mucosal layer, or with superficial submucosal invasion, have a very low risk of lymph node metastases [9]. Endoscopic mucosal resection (EMR) is a technique widely used in Western countries for treatment of BE with HGD and early EAC, due to its safety profile, low cost and rapid recovery. The most commonly employed techniques are cap-assisted technique and multiband ligation-assisted technique [10]. EMR is limited with respect to the re- 
section size, and as a result, larger lesions can only be resected in piecemeal fashion which has been associated with higher local recurrence rates. Furthermore, piecemeal lesion removal impedes the accurate histopathologic evaluation of completeness of the resection (e.g. R0 resection) [11].

The inherited shortcomings of EMR prompted the development of endoscopic submucosal dissection (ESD) which was introduced in Japan as therapy for early gastric cancer. ESD carries a number of advantages over EMR. It provides en-bloc resection of dysplastic lesions regardless of size which allows accurate histologic evaluation of both deep and lateral margin of the resected specimen $[12,23]$. The overwhelmingly positive results with gastric ESD in Japan quickly allowed for the extension of this technique as a therapy for early squamous cell cancer (SCC) of the esophagus with reported en-bloc resection rate from 95 to $100 \%$ with associated very low recurrence rates $[14,15]$. In contrast, data on esophageal ESD as a therapy for BE and early EAC are limited and consist largely of relatively small retrospective case series [16-20]. Only two prospective studies were published in Europe consisting of 55 and 87 patients with early EAC [21,22]. Therefore, the aim of this study was to prospectively evaluate the technical success and adverse events of ESD in patients with BE with HGD or early EAC as performed in a Western tertiary referral center.

\section{Patients and methods \\ V}

\section{Patients}

This is a prospective cohort study of patients diagnosed with $\mathrm{BE}$ and HGD/early EAC who underwent ESD in our center from October 2013 to July 2015 . The study protocol was approved by the institutional review board (IRB) in our institution and all patients signed research informed consent.

Indications for ESD included: (1) BE with early EAC defined as lesions with intramucosal cancer or estimated superficial submucosal invasion; (2) Early EAC with positive lateral margin after EMR; and (3) BE with area of nodularity with HGD that could not be removed en-bloc with EMR.

\section{ESD Equipment and Procedures}

The extent and type of lesion were evaluated with white light and narrow band imaging (NBI) endoscopy during initial planning upper endoscopy and with endoscopic ultrasound (EUS). The circumferential and maximum extent of BE were described according to Prague classification [23]. The size and macroscopic appearance of the target lesion was classified according to the Paris classification [24]. Pre-ESD biopsy was available in all cases. During the same session, EUS was performed using the GF-UE160 radial scanning echoendoscope (Olympus America, Center Valley, PA, USA). The EUS examination was aimed at excluding more advanced disease (e.g. T2) or lymph node metastasis.

At a separate session, ESD was carried out by a single endoscopist (PVD). These were the first patients with BE treated with ESD at our institution, but that followed a period of intensive hands-on training in the technique. All cases were performed under general anesthesia with endotracheal intubation with the patient in left lateral decubitus position. Antibiotic prophylaxis was not used. The GIF-HQ 190 upper endoscope (Olympus America, Center Valley, PA, USA) fitted with distal attachment(D-201-11804, Olympus America, Center Valley, PA, USA) was used in all cases along with the VIO 300 D electrosurgical unit (ERBE USA, Marietta, GA, USA).
ESD was performed using the following steps. First markings were placed with the Dual knife (KD-650, Olympus America, Center Valley, PA, USA) $5 \mathrm{~mm}$ from the margin of the lesion as determined with white light and NBI endoscopy. Next a mixture of saline/hydroxymethyl cellulose/indigo carmine was injected into the submucosal space at the distal aspect of the lesion. A mucosal precut then was made using the Dual knife. A full circumferential incision was then made with the IT nano (KD-612, Olympus America, Center Valley, PA, USA) after repeat injections in the submucosal space. Finally, submucosal dissection was carried out using IT nano knife, supplemented by use of the Dual knife in areas with submucosal fibrosis.

After retrieval, each specimen was pinned immediately on corkboard. Specimen size was recorded and photographs were taken of it before it was sent for processing.

\section{Post-procedure management and follow up}

The decision to admit the patient was left to the discretion of the endoscopist, but in general, patients who had experienced adverse events, had multiple comorbidities, or had a prolonged procedure were admitted for observation. Otherwise, patients were discharged home. All patients were prescribed an oral proton pump inhibitor (PPI) twice daily with the recommendation to continue this therapy indefinitely. The timing of follow-up endoscopy was established based on the extent of the resected area and histologic evaluation. For resections of greater than $50 \%$ of the esophageal circumference, follow-up endoscopy was schedule at 4 to 6 weeks post-ESD in an attempt to detect early any esophageal strictures and initiate endoscopic dilations. For patients whose lesions had negative margins (R0 resection) and favorable features (well differentiated, no submucosal invasion, no lymphatic and vascular invasion), follow-up endoscopy was performed at 3 to 4 months unless earlier endoscopy was indicated based on the extent of the resection (as per \#1 above).

When necessary, patients underwent subsequent radiofrequency ablation of residual flat BE. When the ESD was considered noncurative as detailed below, patients were referred for evaluation for surgical resection.

\section{Study outcomes and definitions}

The main study outcomes were en-bloc and complete (e.g. R0) resection. Secondary outcomes included: curative resection rate, lesion removal time, resection speed, correlation between preand post-ESD histologic findings, and adverse events.

En-bloc resection was defined as successful removal of the lesion in one piece. R0 (complete) resection was defined as en-bloc resection with histological margins (lateral and deep) free of tumor involvement.

Curative resection was defined as R0 resection with any of the following findings: (a) intramucosal cancer, differentiated type, no lymphatic and vascular invasion, and no ulceration, irrespective of tumor size; (b) intramucosal cancer, differentiated type, no lymphatic and vascular invasion, and tumor less than $3 \mathrm{~cm}$ in size, irrespective of ulceration findings; (c) superficial submucosal cancer invasion within $500 \mu \mathrm{m}$, differentiated type, no lymphatic and vascular invasion; or (d) high-grade dysplasia. If present, submucosal fibrosis was graded as mild, moderate or severe, as previously described [25].

Adverse events (AEs) were defined according to the American Society of Gastrointestinal Endoscopy position statement [26]. Esophageal stricture was defined as narrowing severe enough to cause symptoms and require endoscopic dilation. 


\section{Results}

\section{$\nabla$}

\section{Patient characteristics}

The study included 36 consecutive patients. The characteristics of the patients included in this study are summarized in $\bullet$ Table 1. Median age was 69 (range 42 to 80 ). There were 32 men and four women. Fourteen patients (39\%) had received prior therapy for underlying BE. Four had underdone EMR only while the other 10 had received a combination of APC, EMR and cryotherapy.

\section{Lesion characteristics}

The majority of the lesions (61\%) were located in the distal esophagus immediately proximal to the GE junction ( $\bullet$ Table 1 ). Most of the lesions were found in the background of the short segment $\mathrm{BE}(\mathrm{C} 0-1 \mathrm{M} 1-2)$. Less than one-third of patient had long-segment BE with maximal extent up to $11 \mathrm{~cm}$. The main lesion type, based on Paris endoscopic classification, was 0-Ila found in more than half the lesions (56\%) [24]. The least common was type 0 -III with only one lesion encountered during this study. Eight patients (22\%) had polypoid ( $0-$ Ip) lesions.

\section{ESD outcomes}

En-bloc resection was achieved in all cases (100\%) ( $\bullet$ Table2). Median procedure time was 88 minutes, with the shortest procedure being approximately 30 minutes while almost 6 hours were needed for removal of a $13-\mathrm{cm}$ long-segment circumferential lesion. The median maximal diameter of resected specimens was $49 \mathrm{~mm}$. All removed specimens involved at least $40 \%$ of the circumference, while complete circumferential 360-degree dissection was performed in five patients. The majority of the lesions $(58 \%)$ had no underlying submucosal fibrosis while seven lesions had severe or moderate fibrosis. Four of these seven patients had endoscopic treatment of BE, including EMR, RFA, and/or cryotherapy prior to ESD. Of note, while underlying fibrosis might increase the length of the procedure, it did not preclude en-bloc lesion resection.

Complete resection of the tumor (e.g. all margins negative, R0 resection) was accomplished in 29 patients (81\%) ( $\odot$ Table 3). Seven patients (19\%) had incomplete resection (R1 resection). In all seven patients tumor was present at the deep margin. In addition, four of the seven patients had tumor invasion at the lateral margin as well. The overall curative resection rate was $69 \%$.

\section{Histopathology}

Intramucosal EAC was found in 13 patients (36\%), while submucosal invasion was present in 13 patients (36\%). Two tumors were multifocal and one specimen had no discreet lesions. Nine patients (25\%) had long-segment BE with multifocal HGD.

The biopsy specimens prior to ESD matched the ESD histopathological findings in only 15 cases (41\%). Fifteen pathology reports prior to ESD showed HGD (with four cases suspicious but not definitive for adenocarcinoma) while the actual ESD specimens revealed five intramucosal EACs and five carcinomas with submucosal invasion. Five cases were referred for intramucosal carcinoma on surveillance BE biopsies but ESD specimens revealed HGD. One case of adenocarcinoma found to have invasive features on pre-ESD biopsies was classified as intramucosal after ESD, while five intramucosal carcinomas were found to have submucosal invasion after complete resection by ESD ( $\bullet$ Table 4$)$.

\begin{tabular}{|c|c|}
\hline \multicolumn{2}{|l|}{ Clinical characteristics } \\
\hline Number of patients ( $n$ ) & 36 \\
\hline Female/male & $4 / 32$ \\
\hline Age, median (range), years & $69(42-80)$ \\
\hline \multicolumn{2}{|l|}{ Prior treatment, $\mathrm{n}(\%)$} \\
\hline EMR only & $4(11)$ \\
\hline Combination (EMR, APC, cryotherapy) & $10(28)$ \\
\hline \multicolumn{2}{|l|}{ Lesion characteristics } \\
\hline \multicolumn{2}{|l|}{ Location, n (\%) } \\
\hline Esophageal body & $14(39)$ \\
\hline Gastroesophageal junction & $22(61)$ \\
\hline \multicolumn{2}{|l|}{ Barrett's extent, median (range), cm } \\
\hline Circumferential & $0(0-10)$ \\
\hline Maximum & $2(1-11)$ \\
\hline \multicolumn{2}{|c|}{ Macroscopic type (Paris classification), n (\%) } \\
\hline Ip & $8(22)$ \\
\hline lia & $20(56)$ \\
\hline lib & $2(6)$ \\
\hline Mixed & $5(14)$ \\
\hline III & $1(2)$ \\
\hline
\end{tabular}

EMR, endoscopic submucosal resection; APC, argon plasma coagulation

\begin{tabular}{|c|c|}
\hline En-bloc resection & $36(100 \%)$ \\
\hline Procedure duration, median (range), min & $88(34-358)$ \\
\hline Maximum specimen diameter, median (range), mm & $49(21-125)$ \\
\hline Resected area, median, $\mathrm{mm}^{2}$ & 1673 \\
\hline \multicolumn{2}{|l|}{ Extent of resection, $n(\%)$} \\
\hline Less than $25 \%$ & 0 \\
\hline $25-50 \%$ & $16(44)$ \\
\hline $51-75 \%$ & $13(36)$ \\
\hline More than $75 \%$ & $7(20)$ \\
\hline \multicolumn{2}{|l|}{ Submucosal fibrosis, n (\%) } \\
\hline None & $21(58)$ \\
\hline Mild & $8(22)$ \\
\hline Moderate & $4(11)$ \\
\hline Severe & $3(8)$ \\
\hline Adverse events - total, $\mathrm{n}(\%)$ & $8(22)$ \\
\hline \multicolumn{2}{|l|}{ Early (within 24 hours), n (\%) } \\
\hline Bleeding & $1(3)$ \\
\hline Perforation & 0 \\
\hline \multicolumn{2}{|l|}{ Late } \\
\hline Bleeding & 0 \\
\hline Stenosis & $7(19)$ \\
\hline Perforation & 0 \\
\hline
\end{tabular}

\section{Adverse events}

AEs occurred in six patients (22\%) ( $\bullet$ Table 2$)$. In one patient, post-procedural bleeding developed within 24 hours. Re-look endoscopy showed a bleeding vessel, which was treated with dual therapy (injection with epinephrine and electrocautery with bipolar probe). No blood transfusions were required. Esophageal strictures were the only delayed complications and occurred in seven patients (19\%). Four patients developed moderate stenosis which required one to four serial dilations. One patient with long $(125 \mathrm{~mm})$ circumferential submucosal dissection developed refractory stenosis. This was managed with placement of two overlapping fully covered Endomaxx (Merit Medical, Salt Lake City, UT, USA) esophageal stents to bridge the mucosal defect immediately after the procedure followed by multiple dilations (a total of 15 dilations within 8 months following the ESD). 
Table 3 Histopathologic evaluation.

\begin{tabular}{|c|c|}
\hline R0 resection, $\mathrm{n}(\%)$ & $29(81 \%)$ \\
\hline \multicolumn{2}{|l|}{ Incomplete resection, n (\%) } \\
\hline At lateral margin & 0 \\
\hline At deep margin & $3(8)$ \\
\hline At both margins & $4(11)$ \\
\hline \multicolumn{2}{|l|}{ Pathology, n (\%) } \\
\hline High-grade dysplasia & $10(28)$ \\
\hline Intramucosal adenocarcinoma & $13(36)$ \\
\hline Invasive adenocarcinoma & $13(36)$ \\
\hline \multicolumn{2}{|l|}{ Histological grade, $n$} \\
\hline G1 & 10 \\
\hline G2 & 11 \\
\hline G3 & 3 \\
\hline Gx & 3 \\
\hline \multicolumn{2}{|l|}{ Depth of invasion, $\mathrm{n}$} \\
\hline pT1a & 13 \\
\hline pT1b & 10 \\
\hline pT2 & 3 \\
\hline Lymphatic and vascular invasion, $\mathrm{n}$ & 8 \\
\hline
\end{tabular}

Gx, grade cannot be assessed

This patient currently is asymptomatic without a stent and has not required any further dilation for more than 8 months. There were no perforations and no ESD-related mortality.

\section{Follow up}

Most of the patients (75\%) were discharged to home in the same day after a short observation in the recovery area. The decision to admit the patient was left at the discretion of the endoscopist.

Median follow up for patient who underwent curative endoscopic resection was 10 months (range 1-23 months). There was no local recurrence (as documented on biopsies from the resection site) or metastatic disease in these patients.

Ten patients (31\%) underwent surgical resection (esophagectomy). The main indication for esophagectomy was R1 (incomplete) resection in six patients. The other four patients had R0 resection but the resection was considered non-curative due to the presence of lymphatic or vascular involvement. Information on follow up after surgery was available for seven of these patients, while three patients elected to have surgery at another facility and were lost to follow up. Three patients had lymph node involvement in the surgical specimen. All the others showed no remaining disease and no lymph node involvement.

Subsequent endoscopic treatments (RFA) to remove residual flat $\mathrm{BE}$ were administered during the follow-up period in five patients.

\section{Discussion \\ $\nabla$}

ESD has become the treatment of choice in early SCC in Japan [14]. Only limited retrospective reports for ESD of EAC are available $[17,27,28]$. Omae et al. reported en-bloc and curative resection rates of $100 \%$ and $84 \%$, respectively, with no recurrent or metastatic carcinoma detected during a mean follow-up period of 33 months [27]. Kagemoto reported similar results in a cohort of 23 patients [17]. Interestingly, Hoteya et al., in a large study of 1224 patients that included 25 cases of EAC, found that the rates for curative resection were significantly lower when compared with junctional and non-junctional gastric cancers [28]. It is worth mentioning that while there are well-established criteria for ESD for SCC, despite these promising results, there are still no criteria for endoscopic therapy for selecting patients for endoscopic therapy early EAC [17].

In Western countries, ESD expertise remains limited to several centers and only a few endoscopists take on the challenge of performing ESD in a difficult location such as the distal esophagus and gastroesophageal junction (GEJ). Farhat et al., based on a survey on the emerging practice of ESD in France, reports $88.9 \%$ enbloc and $77.8 \% \mathrm{R} 0$ resection rates of esophageal lesions [29]. Chevaux et al. describes a retrospective study in a larger cohort of 75 patients with rates of en-bloc resection rate of $90 \%$ and curative resection of neoplasia of 56\% [21]. Neuhaus et al., in a small prospective study, reported a favorable en-bloc resection rate of $90 \%$ but only a $38.5 \%$ R0 resection rate [16]. Probst et al. reports a high en-bloc resection rate of $95.4 \%$ for EAC with a curative resection rate of $72.4 \%$ [22]. In our center, the primary endoscopist, who was already highly experienced, underwent a prolonged handson training program in ESD prior to initiating the procedure at our institution [30]. These data demonstrate the feasibility of this approach for centers without extensive previous gastric ESD experience as it is typically recommended in Japan.

Our prospective study highlights several important findings:

1. En-bloc resection can be accomplished in the vast majority of Barrett's lesions (100\% in our series).

2. Complete resection ( $\mathrm{R} 0$ resection) can be achieved in patients with $\mathrm{BE}$ at a rate that is comparable with $\mathrm{R} 0$ resection rates reported for other locations and types of lesions. In our study, the R0 and curative resection rates were $81 \%$ and $69 \%$, respectively, which is on the upper end of reported rates in SCC of the esophagus, early gastric cancer and colonic lesions $[19,20,27,31,32]$.

3 . The number of incomplete (R1) resections in our series appears to be a consequence of tumor presence at the deep margins. All seven cases of incomplete resection had deep margin involvement. Furthermore, four of the seven patients with deep margin involvement also had a positive lateral margin. This issue may be specific to BE where the sub-squamous extension of metaplastic epithelium is a recognized but not well

Table4 Discrepancies between pre- and post-ESD histopathologic evaluation.

\begin{tabular}{|c|c|c|c|c|}
\hline & & \multicolumn{3}{|l|}{ Pre-ESD biopsy } \\
\hline & & High-grade dysplasia & Intramucosal EAC & $\begin{array}{l}\text { Invasive EAC with } \\
\text { submucosal involvement }\end{array}$ \\
\hline \multirow[t]{3}{*}{ Post-ESD } & High-grade dysplasia & 5 & 5 & 0 \\
\hline & Intramucosal EAC & 5 & 7 & 1 \\
\hline & Invasive AC with submucosal involvement & 5 & 5 & 3 \\
\hline
\end{tabular}

ESD, endoscopic submucosal dissection; EAC early esophageal adenocarcinoma; AC, adenocarcinoma 


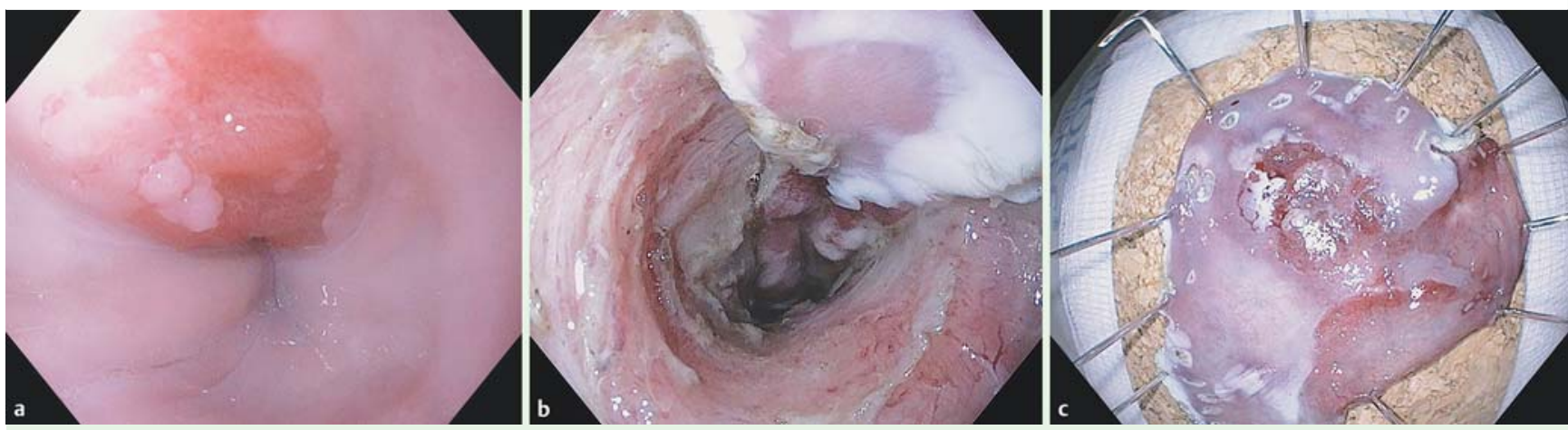

Fig. 1 Endoscopic submucosal dissection with curative resection of Barrett's esophagus in a 64-year-old male. a Endoscopic view of C0M2 Barrett's esophagus with a $1-\mathrm{cm}$ lesion, Paris classification 0 - Ila + Ilc. b Resection area after dissection and hemostasis. c Resected specimen with at least 10 -mm safety margin around the suspected neoplastic lesions.

publicized phenomenon [28]. As a result of our findings, we currently allow for at least a 10 -mm distance between the visible margin of the tumor and the circumferential incision (๑ Fig. 1). However, we recognize that this technique might result in longer procedure times and higher stricture rates due to the wider submucosal resection.

4. In a significant majority of patients (59), there was a discrepancy between the pre- and post-ESD histopathological diagnosis. As such, ESD provided far more accurate pathologic evaluation which allowed for better informed follow-up decisions. Although curative resection remains the main goal of ESD, based on our findings, it can also be an important staging tool. The importance of accurate histopathologic diagnosis cannot be overstated. An inaccurate diagnosis could have substantial deleterious consequences. A patient with benign disease could undergo esophagectomy while a patient with submucosal or lymphatic or vascular invasion could be treated endoscopically instead of with surgical removal. Several studies describe the risk of inaccurate diagnosis by forceps biopsy and upgrading of lesions once removal was done with endoscopic resection. Most of the studies focused on gastric pathology and reported rates of histologic discrepancy between the initial biopsy and resected specimen as high as $50 \%$ [3335]. Our study is the first one to show significant discrepancy between the histological diagnosis at the initial biopsy and the actual ESD specimen in BE patients.

5. Interestingly, our cohort of patients included a significant proportion with advanced EAC diagnosed on the ESD specimens (36\%). Ten of these thirteen patients were under-diagnosed with HGD and/or intramucosal EAC on pre-ESD samples obtained with biopsy forceps. The remaining three patients who had invasive EAC on pre-ESD biopsy samples underwent ESD either because of patient preference or significant underlying comorbidities that conferred a high surgical risk.

6. ESD in BE can be accomplished with an acceptable procedure time. In our study, the median procedure time was 88 minutes, which is shorter than most reported studies on esophageal ESD for comparable size lesions $[17,18,21]$. As anticipated, the median procedure time was shorter for lesions located in the body (77 minutes) compared with that for lesions located at the GEJ (93.5 minutes). This is likely due to the more difficult lesion location.

7. ESD in BE can be safely performed and severe AEs are unusual. We did not experience any perforations or severe bleeding. The rate of esophageal stricture formation was acceptable
( $19 \%$ in our study) and was related to the extent of the resection. Currently, there are no widely acceptable criteria/protocols for management of post-ESD stricture. Approaches used to decrease the risk of stricture formation include oral or topical steroids, stent placement and serial dilations [36,37]. In our study, $83 \%$ of patients had submucosal dissection involving $50 \%$ or more of the esophageal circumference. Unsurprisingly, in our series two-thirds of patients who developed stenosis had resection of $80 \%$ or more of the luminal circumference. Importantly, all of the strictures were easily treated with endoscopic dilation except in one patient who developed severe stricture. That patient had a $125-\mathrm{mm}$ long segment of $\mathrm{BE}$ with multifocal intramucosal carcinoma. Therefore a full 360degree 125-mm long circumferential ESD was carried out. The patient required multiple dilations and stent placement but ultimately the stricture resolved and currently the patient has not required any further dilation for more than 8 months. Our data mirror the findings in esophageal ESD for SCC with a reported rate of stricture formation in $90 \%$ of patients with resection involving more than $75 \%$ circumference [38]. Unfortunately, our study does not provide insight on which stricture-preventing strategy works best. Currently, we use the following protocol for mucosal resection $>50 \%$ of the esophageal lumen circumference based on theoretical considerations and risk/benefit assessment: 1) oral PPI twice a day indefinitely; 2) topical steroid (fluticasone $220 \mathrm{mcg}$ ) to swallow twice a day for 2 months; and 3) EGD 2 weeks after the ESD in order to detect any strictures early and initiate dilation protocol.

8. Most patients can be safely discharged the day of $\operatorname{ESD}$ (75\% in our series). Only one patient, who had undergone resection of an early EAC at GEJ (resected specimen size $42 \times 24 \mathrm{~mm}$ ), returned within 24 hours with hematemesis. At presentation, he had stable vital signs and a drop of 1 unit hemoglobin from his baseline. Urgent EGD showed a non-bleeding esophageal ulcer consistent with recent ESD. No endoscopic intervention was needed but the patient was continued on intravenous PPI for 72 hours.

9. ESD for BE can be successfully and safely introduced in a Western center without extensive previous experience in gastric ESD as recommended in Japan. However, this requires extensive training prior to initiation of the procedure, but it can be done with high success and low complication rates [30,39]. We demonstrated that, contrary to accepted algorithms in the East, performing ESD in BE did not require extensive prior ex- 
perience with ESD in the stomach. This study lends support to the belief that high en-bloc and $\mathrm{R} 0$ resection rates can be achieved with the initial introduction of the ESD program in our Western center.

The validity of our findings is supported by some of the study strengths including: 1) prospective design; 2) well-defined protocol and outcomes; 3) standardized ESD technique; and 4) close and complete follow-up. Our study is not without limitations. Importantly, it has a relatively small sample size.

Nevertheless, to our knowledge, this is the largest prospective study of ESD in BE in the United States. We believe that the carefully collected data from our initial experience and the reported very high en-bloc and $\mathrm{R} 0$ resection rates along with the low rate of AEs will be of value in guiding further patient management and study design. Our single-center/single-endoscopist findings do limit the generalizability of the data but we believe that reporting our encouraging initial results will allow the creation of a multicenter consortium.

In conclusion, our prospective study provides support for the feasibility and safety of ESD for BE with HGD and/or early EAC. ESD can achieve very high en-bloc and R0 resection rates with very low $\mathrm{AE}$ rates in a Western center without extensive previous experience in gastric ESD.

\section{Competing interests: None}

\section{References}

1 Edgren G, Adami HO, Weiderpass $E$ et al. A global assessment of the oesophageal adenocarcinoma epidemic. Gut 2013; 62: 1406-1414

2 Hazelton WD, Curtius K, Inadomi JM et al. The role of gastroesophageal reflux and other factors during progression to esophageal adenocarcinoma. Cancer epidemiology, biomarkers \& prevention: a publication of the American Association for Cancer Research, cosponsored by the American Society of Preventive Oncology 2015: DOI 10.1158/10559965.EPI-15-0323-T

3 Pohl H, Welch HG. The role of overdiagnosis and reclassification in the marked increase of esophageal adenocarcinoma incidence. Journal of the National Cancer Institute 2005; 97: $142-146$

4 de Jonge PJ, van Blankenstein $M$, Looman $C W$ et al. Risk of malignant progression in patients with Barrett's oesophagus: a Dutch nationwide cohort study. Gut 2010; 59: 1030-1036

5 Desai TK, Krishnan K, Samala $N$ et al. The incidence of oesophageal adenocarcinoma in non-dysplastic Barrett's oesophagus: a meta-analysis. Gut 2012; 61: 970-976

6 Murray L, Watson P, Johnston B et al. Risk of adenocarcinoma in Barrett's oesophagus: population based study. BMJ 2003; 327: 534-535

7 Wang KK. Practice Parameters Committee of the American College of Gastroenterology. Updated guidelines 2008 for the diagnosis, surveillance and therapy of Barrett's esophagus. The American journal of gastroenterology 2008; 103: 788 - 797

8 Wani S, Sayana H, Sharma P. Endoscopic eradication of Barrett's esophagus. Gastrointest Endosc 2010; 71: 147-166

9 Zemler B, May A, Ell C et al. Early Barrett's carcinoma: the depth of infiltration of the tumour correlates with the degree of differentiation, the incidence of lymphatic vessel and venous invasion. Virchows Archiv : an international journal of pathology 2010; 456: 609-614

10 Abrams JA, Fedi P, Vakiani E et al. Depth of resection using two different endoscopic mucosal resection techniques. Endoscopy 2008; 40: $395-$ 399

11 Pech O, Behrens A, May A et al. Long-term results and risk factor analysis for recurrence after curative endoscopic therapy in 349 patients with high-grade intraepithelial neoplasia and mucosal adenocarcinoma in Barrett's oesophagus. Gut 2008; 57: 1200-1206

12 Park YM, Cho E, Kang HY et al. The effectiveness and safety of endoscopic submucosal dissection compared with endoscopic mucosal resection for early gastric cancer: a systematic review and metaanalysis. Surg Endosc 2011; 25: 2666-2677
13 Gotoda $T$. Endoscopic resection for premalignant and malignant lesions of the gastrointestinal tract from the esophagus to the colon. Gastrointest Endosc Clin North Am 2008; 18: 435-450, viii

14 Fujishiro M, Kodashima S, Goto $O$ et al. Endoscopic submucosal dissection for esophageal squamous cell neoplasms. Dig Endosc 2009; 21: $109-115$

15 Takahashi H, Arimura Y, Masao H et al. Endoscopic submucosal dissection is superior to conventional endoscopic resection as a curative treatment for early squamous cell carcinoma of the esophagus (with video). Gastrointest Endosc 2010; 72: 255 - 264 e251 - e252

16 Neuhaus H, Terheggen G, Rutz EM et al. Endoscopic submucosal dissection plus radiofrequency ablation of neoplastic Barrett's esophagus. Endoscopy 2012; 44: 1105-1113

17 Kagemoto K, Oka S, Tanaka S et al. Clinical outcomes of endoscopic submucosal dissection for superficial Barrett's adenocarcinoma. Gastrointest Endosc 2014; 80: 239-245

18 Hobel S, Dautel P, Baumbach R et al. Single center experience of endoscopic submucosal dissection (ESD) in early Barrett s adenocarcinoma. Surg Endosc 2015; 29: 1591 - 1597

19 Yoshinaga S, Gotoda T, Kusano C et al. Clinical impact of endoscopic submucosal dissection for superficial adenocarcinoma located at the esophagogastric junction. Gastrointest Endosc 2008; 67: 202 - 209

20 Yamada M, Oda I, Nonaka S et al. Long-term outcome of endoscopic resection of superficial adenocarcinoma of the esophagogastric junction. Endoscopy 2013; 45: 992 - 996

21 Chevaux JB, Piessevaux H, Jouret-Mourin A et al. Clinical outcome in patients treated with endoscopic submucosal dissection for superficial Barrett's neoplasia. Endoscopy 2015; 47: $103-112$

22 Probst $A$, Aust $D$, Märkl B et al. Early esophageal cancer in Europe: endoscopic treatment by endoscopic submucosal dissection. Endoscopy $2014 ; 47: 113-121$

23 Sharma P, Dent J, Armstrong D. The development and validation of an endoscopic grading system for Barrett's esophagus: the Prague C\&M criteria. Gastroenterology 2006; 131: 1392 -1399

24 Endoscopic Classification Review Group. Update on the paris classification of superficial neoplastic lesions in the digestive tract. Endoscopy 2005; 37: $570-578$

25 Jeong JY, Oh YH, Yu YH et al. Does submucosal fibrosis affect the results of endoscopic submucosal dissection of early gastric tumors? Gastrointest Endosc 2012; 76: 59-66

26 Ben-Menachem T, Decker GA. Committee ASoP. et al. Adverse events of upper GI endoscopy. Gastrointest Endosc 2012; 76: 707-718

27 Omae M, Fujisaki J, Horiuchi Yet al. Safety, efficacy, and long-term outcomes for endoscopic submucosal dissection of early esophagogastric junction cancer. Gastric Cancer 2013; 16: 147-154

28 Hoteya S, Matsui A, Iizuka T et al. Comparison of the clinicopathological characteristics and results of endoscopic submucosal dissection for esophagogastric junction and non-junctional cancers. Digestion 2013; 87: 29-33

29 Farhat S, Chaussade S, Ponchon T et al. Endoscopic submucosal dissection in a European setting. A multi-institutional report of a technique in development. Endoscopy 2011; 43: 664-670

30 Draganov PV, Chang M, Coman RM et al. Role of observation of live cases done by Japanese experts in the acquisition of ESD skills by a western endoscopist. World J Gastroenterol 2014; 20: 4675-4680

31 Fujishiro $M$, Yahagi $N$, Kakushima $N$ et al. Endoscopic submucosal dissection of esophageal squamous cell neoplasms. Clin Gastroenterol Hepatol 2006; 4: 688-694

32 Jung da H, Youn YH, Kim JH et al. Endoscopic submucosal dissection for colorectal lateral spreading tumors larger than $10 \mathrm{~cm}$ : is it feasible? Gastrointest Endosc 2015; 81: 614-620

$33 \mathrm{Kim}$ YJ, Park JC, Kim JH et al. Histologic diagnosis based on forceps biopsy is not adequate for determining endoscopic treatment of gastric adenomatous lesions. Endoscopy 2010; 42: 620-626

34 Won CS, Cho MY, Kim HS et al. Upgrade of lesions initially diagnosed as low-grade gastric dysplasia upon forceps biopsy following endoscopic resection. Gut Liver 2011; 5: 187-193

35 Takao M, Kakushima N, Takizawa Ket al. Discrepancies in histologic diagnoses of early gastric cancer between biopsy and endoscopic mucosal resection specimens. Gastric Cance 2012; 15: 91 - 96

36 Sato H, Inoue H, Kobayashi Y et al. Control of severe strictures after circumferential endoscopic submucosal dissection for esophageal carcinoma: oral steroid therapy with balloon dilation or balloon dilation alone. Gastrointest Endosc 2013; 78: 250-257 
37 Yamaguchi N, Isomoto H, Nakayama T et al. Usefulness of oral prednisolone in the treatment of esophageal stricture after endoscopic submucosal dissection for superficial esophageal squamous cell carcinoma. Gastrointest Endosc 2011; 73: 1115-1121
38 Ono S, Fujishiro M, Niimi K et al. Predictors of postoperative stricture after esophageal endoscopic submucosal dissection for superficial squamous cell neoplasms. Endoscopy 2009; 41: 661-665

39 Coman RM, Gotoda T, Draganov PV. Training in endoscopic submucosal dissection. World J Gastrointest Endosc 2013; 5: 369-378 